\title{
Editorial to the Topical Collection on Star Formation
}

\author{
A.M. Bykov ${ }^{1}$ - C. Charbonnel ${ }^{2}$ - P. Hennebelle ${ }^{3}$. \\ A. Marcowith ${ }^{4}$ - G. Meynet ${ }^{5} \cdot$ M. Falanga ${ }^{6}$. \\ R. von Steiger 6
}

Published online: 11 May 2020

(C) Springer Nature B.V. 2020

Formation of stars at different evolution stages of the Universe is at the heart of the natural ranging from cosmogony to life origin studies. Indeed stars are the very fundamental building blocks of the Universe, which strongly influence and to some extent, control galaxy and planet formation and evolution. This topical collection is devoted to an in-depth examination of complex astrophysical phenomena of star formation via multi-wavelength observations and modeling. From their birth to their death, stars are playing a major role in chemical evolution of the matter and in the energy budget of galaxies via their radiation, their winds, and for the most massive ones via their explosion into supernovae. The first stars have provided the seeds of supermassive black holes with fundamental implications for the evolution of the observable Universe. The complex interplay of physical processes from gas and dust dynamics and cosmochemistry to nuclear physics leading to formation of protostars and protoclusters inside molecular clouds followed by their MHD and radiative feedback on the clouds are currently subjects of a great interest. Despite a lot of progress of the sensitive multi-wavelength observations and high performance modeling, a number of

Star Formation

Edited by Andrei Bykov, Corinne Charbonnel, Patrick Hennebelle, Alexandre Marcowith, Georges Meynet, Maurizio Falanga and Rudolf von Steiger

\footnotetext{
$\triangle$ A.M. Bykov

byk@astro.ioffe.ru

1 Ioffe Institute, 194021, St. Petersburg, Russian Federation

2 Department of Astronomy, University of Geneva, Chemin de Pegase 51, 1290 Versoix, Switzerland

3 AIM/CEA/CNRS, Université Paris-Saclay, Université Paris Diderot, Sorbonne Paris Cité, 91191 Gif sur Yvette, France

4 Laboratoire Univers et Particules de Montpellier (LUPM), Université de Montpellier, CNRS/IN2P3, CC72, place Eugène Bataillon, 34095 Montpellier Cedex 5, France

5 Observatoire de Geneve, Department of Astronomy of Geneva University, 51 chemin des Maillettes, 1290 Sauverny, Switzerland

6 International Space Science Institute, Bern, Hallerstrasse 63012 Bern, Switzerland
} 
fundamental issues remain enigmatic as of yet. This is because the star formation process has a multi-scale nature with a strong non-linear feedback from radiation, MHD flows and non-thermal high energy particles. Magnetic fields, dust kinetics, as well as a number of non-thermal plasma processes play a role in evolution and dynamics of star-forming regions as we observe it from radio bands to gamma-rays.

The amount of data on star formation obtained recently with the Herschel, HST, Gaia, XMM-Newton, Chandra and Fermi space telescopes, as well as with the ground based LOFAR, VLBI, ALMA, VLT and H.E.S.S. observatories is growing fast. These missions have produced vast and high-quality data bases. The forthcoming missions ATHENA, JWST, SPICA, EUCLID and others will uncover new phenomena in star formation.

The goal of this topical collection is to review the major processes governing star formation and to investigate how they are interlinked. This volume provides an in-depth review of the tremendous theoretical and observational progress that has been made in the recent past and outline some perspectives.

Among the fundamental issues discussed are: The role of gravity in formation and evolution of molecular clouds. The nature of supersonic and magnetized turbulence in giant molecular clouds. The role of stellar feedback (supernovae, HII regions, winds) in regulating star formation. Radiation transfer, magnetic fields and gas ionization by cosmic rays in star-forming regions. The role of radiation feedback, jets and magnetic fields in stellar clusters. The origin of the stellar initial mass function and how universal it is across various environments. The origin of the first stars and the star formation rate across the cosmic evolution. These and other aspects of observations and physics of star formation, as well as related phenomena are presented in this topical collection.

Star formation involves multi-scale time-dependent interactions between a great variety of sub-systems. Each feedback loop has some specific physical processes. The reviews in this topical collection scans these processes starting from observations and then focuses on the essential thermal and chemical mechanisms at work in star forming regions.

\section{The Physics of Star Formation: Basic Processes}

The review by Philip Girichidis et al. commences with a description of the main properties and the scaling laws which rule the star-formation rates in galaxies. It continues with a description of the main properties of interstellar gas and dust. Then it addresses the $\mathrm{H}_{2}$ and $\mathrm{CO}$ chemistry in molecular gas as well as the main cooling and heating mechanisms. The review then focuses on the thermodynamical properties of the interstellar medium: the thermal balance and the thermal instability. The review has a dedicated section to three main components which control the molecular cloud life-cycle, namely the turbulence, the magnetic fields and the gravitation. The review finishes by a survey of the different feedback processes at work in star formation, processes further detailed in other reviews part of this topical collection.

\section{Molecular Cloud Life Cycle}

Giant molecular clouds (GMC) are the building blocks of galaxies and as such they detain several clues of the star-formation process. In particular, questions such as their life time and star-formation efficiency, that is to say the typical fraction of their gas that is eventually turned into stars before they get dispersed are fundamental issues to get a global view of star 
formation within galaxies. The molecular cloud life-cycle with a focus on star formation and their feedback processes is discussed by Mélanie Chevance et al. The various observations that have been obtained in the recent years are reviewed. In particular the recent spatial resolution improvement that came with ALMA has led to tremendous progress as it is now possible to probe the whole GMC populations in several galaxies make it possible to study the link between GMC properties and their environments.

\section{From Diffuse Clouds to Molecular Clouds}

The internal structure of the giant molecular clouds (GMC), where most, if not all, of star formation occurs, is reviewed by Javier Ballesteros-Paredes et al. They present the current knowledge of GMC-formation scenarii and their structure from the galactic scales down to the filament and core scale. The mechanisms of cloud formation from the hot diffuse interstellar medium, the process of molecule formation and the role of the thermal and gravitational instabilities are all discussed. The so-called Larson's relations, describing how mass and velocity dispersion of clumps depend on their size are extensively discussed. Various interpretations of the origin of these relations are discussed. The most recent filament and dense core statistical properties, such as their mass function, recently inferred from the Herschel satellite are presented and discussed in the context of numerical simulations.

\section{The Origin of the Stellar Mass Distribution and Multiplicity}

Given the tremendous role played by stars in the Universe and the strong dependence of their properties on their masses, it is clear that their mass spectrum, the so-called stellar Initial Mass Function (IMF) is one of the most fundamental distribution of the Universe. The fundamental issue of the origin of the stellar mass distribution and multiplicity are discussed in the review by Yueh.-Ning Lee et al. The various models and assumptions which have been proposed and explored are presented. In particular the possible link between the core mass function, that is to say the mass distribution of the pre-stellar dense cores is discussed at length both from an observational and theoretical perspective. The particularly intriguing origin of the peak of the IMF, observed to lie at about 0.3 solar mass is discussed at lengths. Finally, the question of the stellar multiplicity is presented both from an observational and theoretical point of view.

\section{The Collapse: From the Clouds to the Star}

The dense clumps within giant molecular clouds are evolving into the pre-stellar cores and the subsequent gravitational collapse of these cold dusty cores leads to the formation of stars. The review by Anna Rosen et al. review is zooming in to the individual star formation processes, with a broad discussion of both low- and high-mass star formation. The high-mass stars, despite representing only a minor by number population $(\sim 1 \%)$ of the stellar population in star-forming regions, dramatic provide very strong feedback on their natal cloud environments. The ionizing radiation, fast stellar winds, supernova ejecta and cosmic rays produced by massive stars have drastic effect on star and galaxy formation. Low-mass stars are the main stellar constituent of galaxies and also the sites of planet formation. The authors also emphasize the importance of some unresolved issues including the initial magnetic field strengths and orientation, angular momenta, and turbulence of the dense cores from which stars and disks form. 


\section{Formation and Evolution of Disks Around Young Stellar Objects}

A very important issue for star-formation theory is to understand how planet-forming disks are assembled. Indeed, there is more and more evidence that planet formation, which heavily depends on the disk physical properties, begins relatively early after, and maybe during, the time that the star builds its mass. The physics of the formation and evolution of disks around Young Stellar Objects is reviewed in detail in this topical collection by Bo Zhao et al. The recent observations, which to a large extent have revolutionized our knowledge of the planet-forming disks are first reviewed. The theoretical understanding of disk formation is then presented. In particular, the tremendous role played by the magnetic field, which largely controls the disk formation process, through magnetic braking, is stressed. Recent studies have concluded that non-ideal magnetohydrodynamics becomes very important in this context as it determines the coupling between the gas and the magnetic field and therefore the amount of magnetic braking, that is to say the efficiency of angular-momentum exchange between the disk material and the surrounding envelope. Finally, the associated processes of outflow launching and the formation of multiple systems are discussed as well as their possible implications regarding the properties of protoplanetary disks.

\section{First Stars}

The formation processes of the first stars differ from the present day star formation. Lionel Haemmerlé, L. Mayer, R. Klessen et al. discuss the early phases of star formation during cosmic dawn at very high redshift $(z>20)$. They review the formation at the early epoch metal-free Population III stars, and the first generation of massive black holes (i.e. black hole seeds). The impact of early accreting massive black holes as well as the early star-forming galaxies on the reionization of the Universe is outlined. The early production, propagation, and absorption of $\mathrm{LyC}$ radiation results in reionization of hydrogen during the first billion years of the cosmic history, while the full reionization of helium is argued to have occurred at a later time, when bright UV-emitting AGNs became sufficiently abundant.

\section{Star Clusters}

Star clusters are key constituents of the complex galactic ecosystem. Observed properties of cluster populations in the local Universe are reviewed by Angela Adamo, P. Zeidler, D. Kruijssen et al. The dense, hierarchically structured collapsing gas clouds are the birth places of the star clusters. The efficiency of star formation in the compact regions is expected to be high. The formation and evolution of star clusters are reviewed in this topical collection by Martin Krause, S.R. Offner, C. Charbonnel et al. who argued that stellar birth is a highly dynamical event. Their modeling involves a wide range of scales in space, time, and energy with an important role of stochastic processes across the galaxies. It is argued that just one star formation epoch exists after which the cluster winds remove essentially the gas in cluster.

\section{Cosmic Rays in Small Scale Star Formation}

Production of highly non-thermal particles and radiation play an important role in the evolution of ISM and during the star-formation processes. The Cosmic rays (CRs) in the GeV 
regime energies are among the most important components of the Milky Way balance as their pressure is comparable to those of the thermal gas, magnetic fields and turbulent motions. Being able to penetrate into the dense clumps of molecular clouds, unreachable for the interstellar radiation low energy $\mathrm{CRs},{ }^{1} \mathrm{CRs}$ are often the only gas heating and ionisation agent in the starforming regions. The acceleration of low-energy CRs by protostellar shocks of the bipolar jets, their propagation and interactions with the matter in the starforming regions is discussed by Marco Padovani, A. Ivlev, D. Galli et al. in this topical collection. While the low-energy CRs accelerated in the protostellar objects are likely not the main contributors to the galactic CR-pool, their role is essential to understand the ionisation rate and the synchrotron emission observed in protostellar environments.

\section{Cosmic Rays and Nonthermal Radiation in Starforming Region}

In a broader galactic context, the star-forming regions may efficiently produce non-thermal radiation, magnetic fields and cosmic rays which play the essential roles in the feedback processes in the galactic environment (especially in starburst galaxies). CRs, their hard radiation and fluctuating magnetic fields are produced in a complicated energetic plasma processes following release of the gravitational energy in both protostellar objects and powerful winds and core collapsed young massive stars. In the review by Andrei Bykov, A. Marcowith, E. Amato et al. in this topical collection, the state-of-the art of the processes of high-energy particle acceleration and radiation in star-forming regions and starburst galaxies is presented. Recent gamma-ray observations provided conclusive evidences that young massive star clusters (e.g. Westerlund 1, 2, Cl*1806-20, RSGC 1 etc.) and the rich OB association Cygnus OB2 are efficient sources of high energy CRs. Theoretical models predicted that these types of sources can accelerate protons up to the $100 \mathrm{PeV}$ energy regime. CRs accelerated in young massive star clusters can help to explain the anomalous ${ }^{22} \mathrm{Ne} /{ }^{20} \mathrm{Ne}$ isotopic ratio observed in low-energy cosmic rays, which is one of the long-standing problems concerning the origin of cosmic rays. Non-thermal radiation from radio to gamma-rays produced in star-forming regions provide unique information about the physical processes of energy release and particle acceleration in star-forming regions; it helps to trace the matter distribution, magnetic fields and interstellar radiation fields in these regions. Non-linear kinetic models describing the evolution of spectra of high-energy particles are reviewed in a connection with the prospects of studies of the non-thermal phenomena in star-forming regions with future multi-messenger observations from radio to gamma-ray energies, gravitational wave and neutrino observatories.

Overall, this collection of reviews provides a timely and comprehensive overview of multi-wavelength observations and theoretical understanding of star formation in the wide astrophysical context. Thus, it will be particularly useful to postgraduate students and researchers active in various areas of astrophysics and space science. This collection is based on an international workshop which took place at ISSI Bern during May 20-24, 2019 where about forty leading experts in star-forming physics discussed the issues presented here.

Publisher's Note Springer Nature remains neutral with regard to jurisdictional claims in published maps and institutional affiliations.

\footnotetext{
${ }^{1}$ That is $\mathrm{CRs}$ with kinetic energies in the range of $\mathrm{MeV}$ to $\mathrm{GeV}$ for protons and $\mathrm{keV}$ to $\mathrm{MeV}$ for electrons. All kinetic energies above are hence defining high energy CRs.
} 